\title{
Opto-electronic pattern recognition system
}

\author{
H. J. TIZIANI, B. H. BEYELER \& W. WITZ \\ Institut fur Technische Physik, ETH-Aussenstation, Hönggerberg, Zurich
}

\begin{abstract}
An opto-electronic technique for the classification of grains of photographic emulsions by shape is described. Fortunately, the shapes to be distinguished differ only in their two-dimensional rotational symmetry group. A special optical filtering procedure has been developed to take account of the implied positional and rotational invariance. The transfer from the optical to the electrical signal is obtained in a simple manner. The method works satisfactorily even for a rather complicated cluster. Some results are discussed.
\end{abstract}

OBJECTIVE CRITERIA for the quality of photographic emulsions are required. The projection of the individual AgBr-crystals of especially prepared emulsions are produced by an electron microscope at a lateral magnification of 10,000 . According to the precipitation conditions, different shapes of crystals occur: cubes, octahedrons and triangular or hexagonal plates. Since the orientation of the crystals is usually such that one side is parallel to the substrate plane, the projections of the crystals are square, rhomboidal, triangular or hexagonal as shown in Fig. 1. This picture represents a mixture of differently precipitated emulsions to show all the characteristic shapes of photographic grains at once. The electron-microscopical preparation has been performed to obtain the projected areas of the grains only. The crystals are sparsely distributed, but some superpositions of two or occasionally more objects forming clusters can occur. The range of variation in the object size of the emulsion to be studied is about $0.05-0.5 \mu \mathrm{m}$.

Manufacturers of photographic emulsions are interested in the statistics of these crystals in shape and size. A method to determine these two parameters automatically must be developed. Measurement of the crystal size to the accuracy required is not too difficult. To investigate the statistics of the shapes is not, however, an easy pattern recognition problem. Position and orientation of the crystals are of no importance and small discrepancies, for instance rounded corners, should be of no significance. Thus the pattern recognition system to be devised must classify the shapes which occur, either separately or in clusters, into four groups independently of position or orientation. Such a system is described here.

\section{POSSIBLE SOLUTIONS}

In principle it is possible to scan the pattern shown in Fig. 1 photoelectrically and to analyse the information in a digital computer. This method, however, needs an exceedingly complicated algorithm.

The reasons for not using the well-known optical filtering techniques such as those proposed by Vander
Lugt, ${ }^{1}$ or Armitage \& Lohmann, ${ }^{2}$ for coherent and incoherent illumination respectively are now briefly discussed. These methods seek correlations between the presented pattern of the unknown material and the filter of the standard comparison object. Such filters are selective with respect to variation of both size and orientation. In this case classification of the objects into the four groups only is required. Nevertheless, it would be possible to employ a large number of rotating filters to account for this size discrepancy. Alternatively, zoom objectives or the method given by Vander Lugt $^{3}$ could also be used. The methods mentioned have not, however, been found to be suitable; they are very sensitive to the accuracy of positioning of the filters and are very slow.

An opto-electronic analogue method was finally adopted with the required invariance properties. The following sections are devoted to the new method. It is assumed that the selection of the cluster or single object is carried out in the object space prior to the filtering procedure.

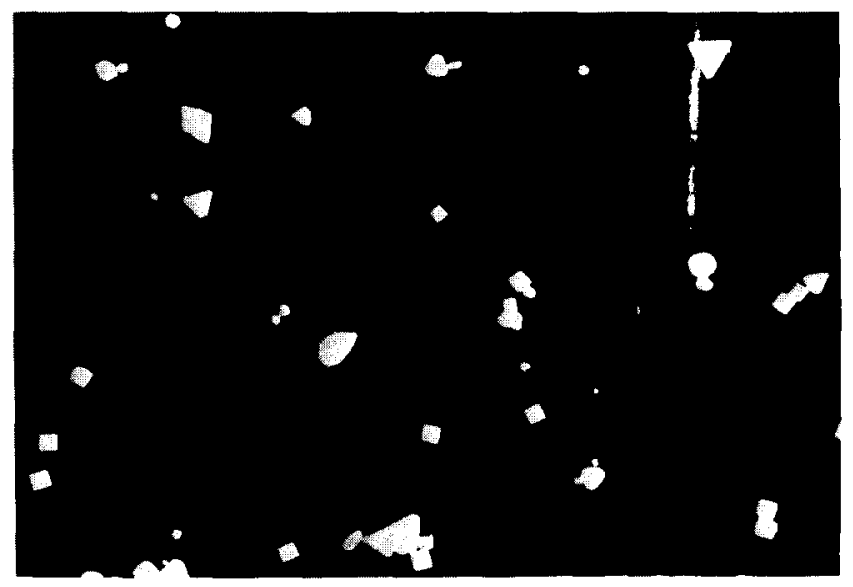

Fig. 1 Example of an electron micrograph 


\section{OPTO-ELECTRONIC PATTERN RECOGNITION SYSTEM}

The two dimensional Fourier transform of the object is obtained in the back focal plane of the lens $L$ shown in Fig. 2. The object in the plane $P_{1}$ is illuminated by a plane wave. The spectrum $a(x, y)$ of the object with the complex amplitude $A(u, v)$ obtained in the back focal plane $P_{2}$ of the lens $L$ is (ref 4)

$$
a(x, y)=\int_{-\infty}^{\infty} A(u, v) \exp [-2 \pi i(u x+v y)] d u d v
$$

$\mathrm{u}$ and $\mathrm{v}$ are the coordinates in the object plane and $\mathrm{x}, \mathrm{y}$ are the normalised coordinates in the back focal plane which are related to the geometrical coordinates $\mathrm{X}, \mathrm{Y}$ in the following way

$$
x=-\frac{X}{\lambda f}, y=-\frac{Y}{\lambda f}
$$

where $\lambda$ is the wavelength of the light and $f$ the focal length.

The intensity in the back focal plane is

$$
\mathrm{i}(\mathrm{x}, \mathrm{y})=|\mathrm{a}(\mathrm{x}, \mathrm{y})|^{2}
$$

and, as is well-known, $i(x, y)$ is invariant on translation of the object in the $(u, v)$ plane.

Typical intensity spectra of examples of each of the four groups are shown in Fig. 3. The rotational symmetry of the corresponding object is manifest in the spectrum. The reason for the three-fold symmetry of the triangular object is explained in the Appendix.

Fig. 3 shows that each of the four groups is distinguished by the azimuth dependence of the energy distribution of the intensity spectrum

$$
e(\Phi)=\int_{0}^{\infty} i(x, y) r d r \text { with } r=\left(x^{2}+y^{2}\right)^{1 / 2}
$$

Except for an energy factor, the function of $e(\Phi)$ is independent of the extent of the object, as indicated in the Appendix. The same conclusions apply with reasonable approximation to the modified function

$$
\mathbf{e}^{\prime}(\Phi)=\int_{\mathbf{r}_{0}}^{\mathbf{r}_{1}} \mathrm{i}(\mathrm{x}, \mathrm{y}) \mathrm{r} \mathrm{dr} \text { with } \mathbf{r}_{1} \gg \mathrm{r}_{0}
$$

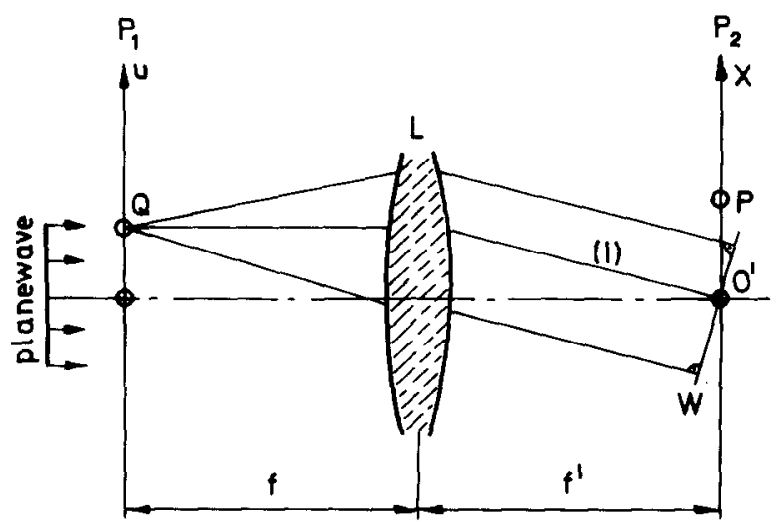

Fig. 2 Fraunhofer diffraction
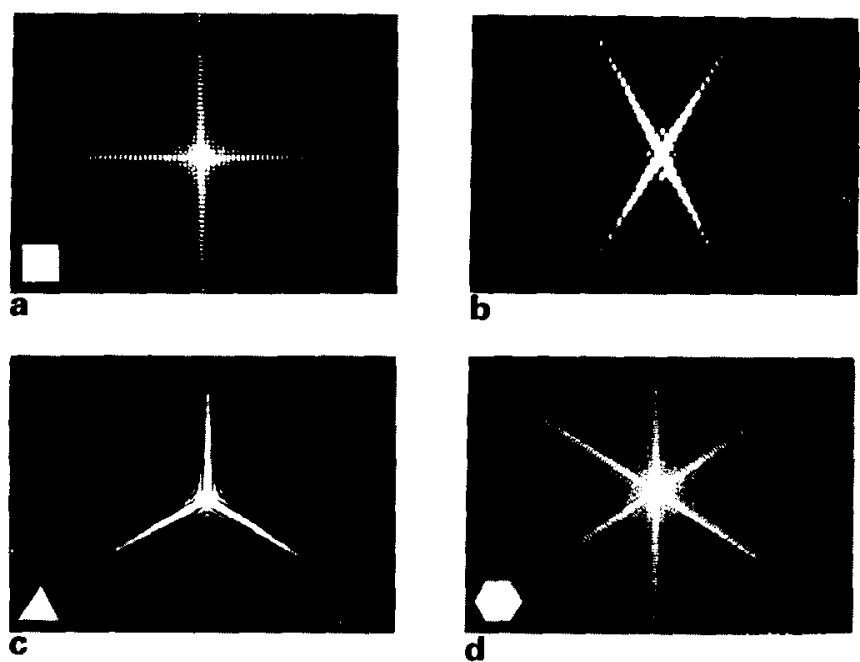

Fig. 3 Typical spectra obtained for each of the four object groups: (a) square, (b) rhombus, (c) triangle, (d) hexagon

where $r_{0}>$ the extent of the zero order of the spectrum of the smallest object to be investigated. The limits of integration are physically reasonable, since the zero order of the spectrum contributes a constant intensity to $e(\Phi)$. Knowing that the central intensity in the spectrum is proportional to the area of the object, this is used for size determination.

\section{REALISATION OF THE METHOD}

The measurement of $e^{\prime}(\Phi)$ is accomplished by rotating a disc $S$ with its centre on the optical axis and with a transparent sector, as shown in Fig. 4. The light flux is collected by the photo-cathode of a photomultiplier behind the rotating disc. The power of the collected light can be written as

$$
f(\Phi)=\int_{\phi-\frac{\Delta \Phi}{2}}^{\phi+\frac{\Delta \Phi}{2}} e^{\prime}(\Phi) d \Phi=e^{\prime}(\Phi)-\star s(\Phi)
$$

where $\mathbf{s}(\phi)$ is the characteristic function of the sector slit, $\Delta \phi$ is the angle of the sector, and $-*$ indicates, as customary, the convolution sign. For an appropriately small angle $\Delta \Phi\left(\Delta \Phi\right.$ of $15^{\circ}$ was found to be adequate for the configuration chosen) there is practically no information lost by considering $f$ rather than $e^{\prime}$.

The behavior of $f(\Phi)$ as a function of the orientation of the object is obvious. Rotations of the objects correspond to the same rotations in the $(x, y)$ plane and hence a phase shift is introduced. It is useful, therefore, to carry out a one-dimensional Fourier transform of $f(\Phi)$. For this reason it seems appropriate to rotate the diaphragm at constant speed. The frequency was chosen to be $\nu_{0}=80$ cycles and, hence, $\Phi=2 \pi \nu_{0} \mathrm{t}$.

This produces an electrical signal of the form

$$
\mathrm{U}(\mathrm{t})=\text { const. } \mathrm{f}\left(2 \pi \nu_{0} \mathrm{t}\right)
$$

from the photomultiplier. 


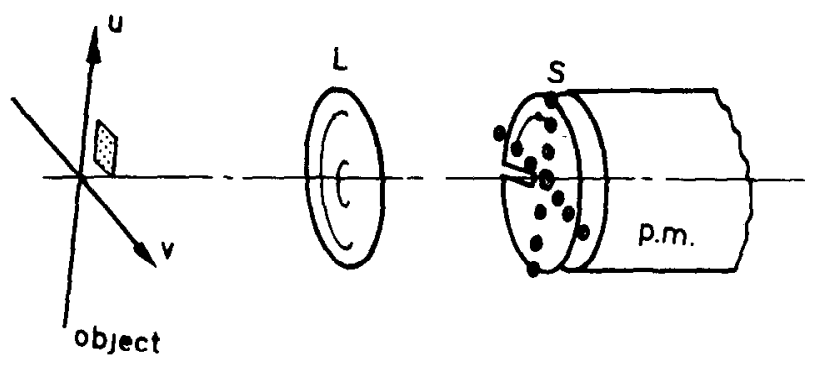

Fig. 4 Schematic of the scanning procedure

Its spcetrum is

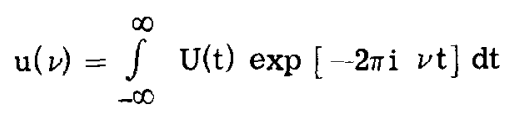

It is obvious that the time-function $U(t)$ is a periodic function and that therefore only spectra with multiple frequencies of $\nu_{0}$ are expected. Thus

$$
\mathrm{u}(\nu)=\sum_{\mathbf{n}=-\infty}^{\infty} \mathrm{c}_{\mathbf{n}} \delta\left(\nu-\mathrm{n} \nu_{0}\right)
$$

A finite integration time introduces a broadening of the spectra, but we are only interested in the ratio of $c_{n}$. Because the $c_{n}$ are the complex Fourier coefficients the moduli of the real function $U(t)$ are related by

$$
\left|c_{n}\right|=\left|c_{-n}\right| \quad n=0,1, \ldots
$$

This is easily verified with the spectrum analyser. A consequence of the rotational symmetry of the objects is the occurrence of coefficients $c_{n}$ listed in Table 1.

\section{Table 1 Complex Fourier coefficients}

\begin{tabular}{lll}
\hline group & object & $\mathrm{c}_{\mathrm{n}} \neq 0$ for $\mathrm{n}=$ \\
\hline $\mathrm{k}=2$ & rhombus & $2,4,6,8,10,12, \ldots$ \\
$\mathrm{k}=3$ & triangle & $3,6,9,12, \ldots$ \\
$\mathrm{k}=4$ & square & $4,8,12, \ldots$ \\
$\mathrm{k}=6$ & hexagon & $6,12, \ldots$ \\
\hline
\end{tabular}

Fig. 5 shows the time function $U(t)$ as well as the frequency spectrum of a sample of each of the four groups. The arrow indicates the position of $\nu_{0}(\mathrm{n}=1)$. It is easily seen that the modulus $\left|c_{n}\right|$ of the coefficients is invariant to rotation of the object; only a constant phase shift is introduced.

All the desirable invariance properties for the coefficients $\left|c_{n}\right|$ are therefore secured. It remains now to investigate the classification into the appropriate group for a known set of coefficients $\left|c_{n}\right|$. Classification of single objects in the four groups could be achieved according to Fig. 5 and Table 1 by considering only the fundamental frequency of the signal. For analysing clusters, however, a set of coefficients $\left|c_{n}\right|$ must be used.

(i) In an ideal system, the coefficients $c_{1}, c_{5}, c_{7}$, $c_{11} \ldots$ would not occur. This is, however, not the case in practice, according to Fig. 5. The reason for the discrepancy is partly due to instrumental error. These coefficients are not carrying useful information and so are neglected in the analysis.

(ii) For $n>12$ the information content of the coefficients is negligible.

The remaining coefficients which agree with Table 1 are

$$
\mathrm{c}_{2}, \mathrm{c}_{3}, \mathrm{c}_{4}, \mathrm{c}_{6}, \mathrm{c}_{8}, \mathrm{c}_{9}, \mathrm{c}_{10}, \mathrm{c}_{12},
$$

This set characterises the pattern in two ways. The length

$$
\mathrm{L}=\left(\sum\left|\mathrm{c}_{\mathrm{n}}\right|^{2}\right)^{1 / 2}
$$

is approximately proportional to the linear dimension of the object (as shown in Appendix) and the direction specifies the shape of the object. From the normalised coefficients $c_{n}^{\prime}=\left|c_{n}\right| / L$, four so-called recognition factors $\mathrm{p}_{\mathrm{k}}(\mathrm{k}=2,3,4$ and 6$)$ may be calculated to obtain an appropriate measure of the group number $\mathrm{k}$. It was found to be adequate to use the linear relation although many other more complicated
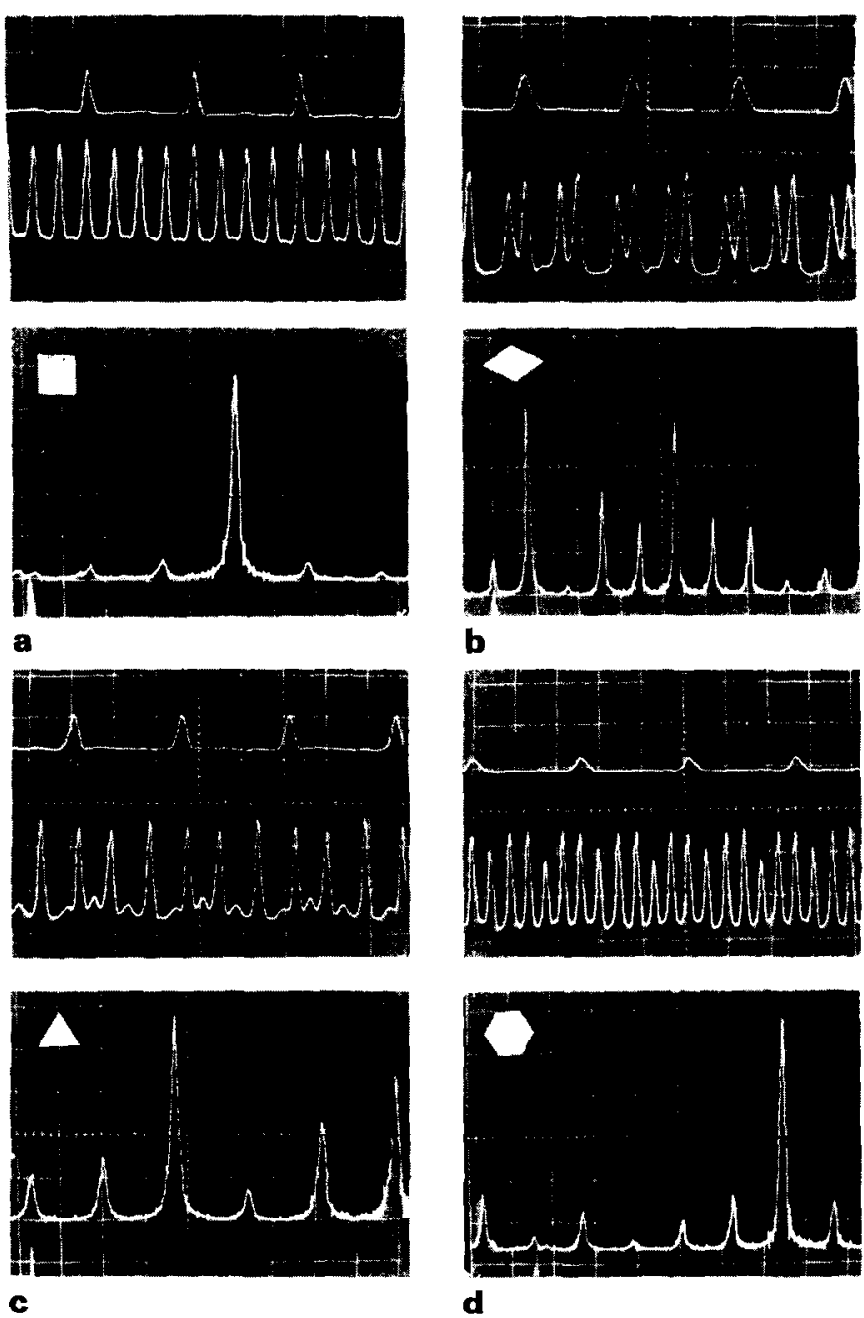

d

Fig. 5 Typical time functions and the corresponding frequency spectra for a sample of each of the four object groups: (a) square, (b) rhombus, (c) triangle, (d) hexagon 


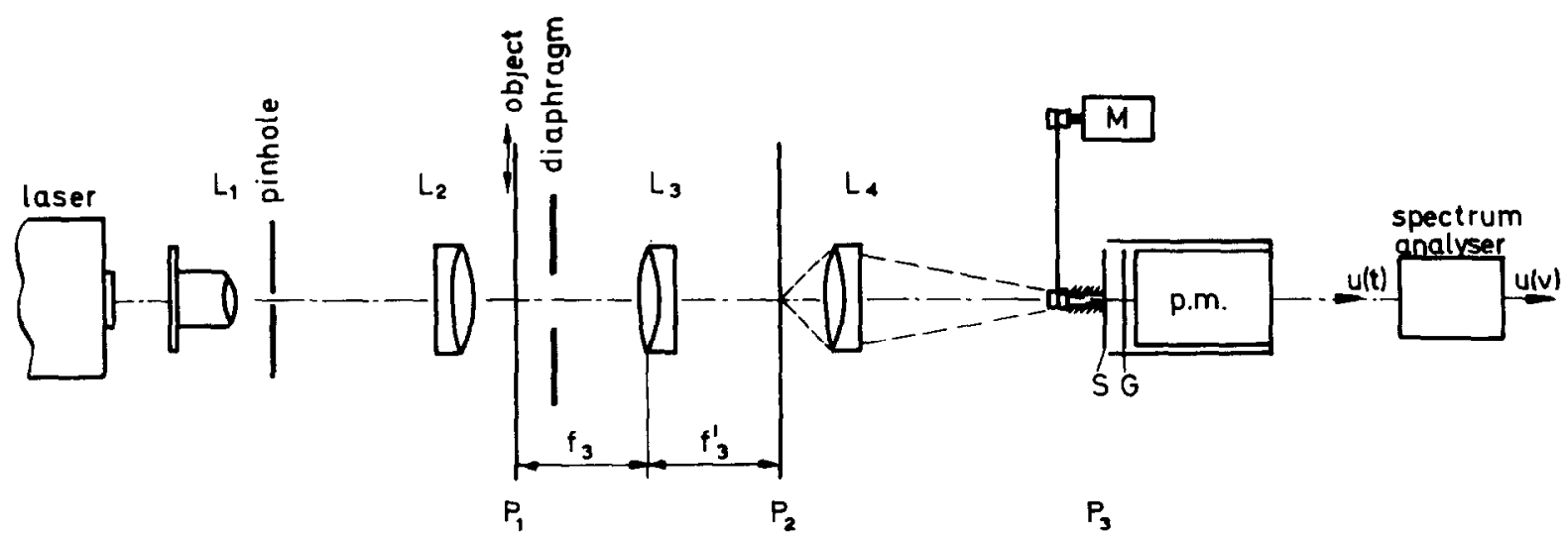

Fig. 6 Schematic of the apparatus

approaches were tried. Hence

$$
\mathrm{p}_{\mathrm{k}}=\sum_{\mathrm{n}} \mathrm{r}_{\mathrm{nk}} \mathrm{c}_{\mathrm{n}^{\prime}}, \quad \mathrm{n}=2,3,4,6,8,9,10,12
$$

The matrix elements $r_{n k}$ were determined empirically and optimised by examining numerous known objects (37), and measuring their coefficients $c_{n}$, so that the $r_{n k}$ could be calculated.

The linear transformation in expression 2 may be carricd out quite casily using an analogue computer (resistance matrix). A set of appropriately tuned. frequency filters may be applied to obtain the coefficients $\left|c_{n}\right|$. At first, these filters were not available, therefore an alternative method of measuring the coefficients on the screen of a spectrum analyser was employed as indicated in Fig. 6.

\section{EXPERIMENTAL PROCEDURE}

The apparatus designed for the experimental investigations is shown schematically in Fig. 6 , where the laser beam is expanded by the lenses $L_{1}$ and $L_{2}$. A plane wave of $\lambda=632.8 \mathrm{~nm}$ illuminates the object in $P_{1}$ and the spectrum is formed by lens $L_{3}$ in its focal plane $P_{2}$. To use a rather short focal length lens $L_{3}$ $(200 \mathrm{~mm})$, an enlarging lens $\mathrm{L}_{4}$ projects the magnified spectrum onto the photocathode of the photomultiplier through the rotating sector slit S. A frequency-stabilised motor $M$ drives the diaphragm. A ground glass $G$ between the diaphragm and photocathode was useful in eliminating the inhomogeneity of the photocathode. The zero order frequency which is not used for shape determination, can be deviated by a small mirror onto a photodiode for size determination. Typical results obtained with this configuration are shown in Fig. 5.

\section{RESULTS}

It should be noticed that for a single and ideal crystal, the recognition factor for the corresponding group is approximately one, but is negligible for the others, provided the normalisation

$$
\mathrm{p}_{\mathrm{k}}^{\prime}=\frac{\mathrm{p}_{\mathrm{k}}}{\left[\Sigma \mathrm{p}^{2} \mathrm{k}\right]^{1 / 2}}
$$

is used.
It was found experimentally that out of 42 single objects, including many with considerably rounded corners, the recognition factor of the associated group was $>0.7$. By contrast the remaining three recognition factors were always $<0.4$. As has already been pointed out, the determination is unaffected by displacement and rotation of the object.

It remains now to investigate the application of the method to the identification of clusters. The theoretical analysis is complex since a number of non-linearities occur.

(i) Even in the object plane $P$, superposition is nonlinear because the common area of two or more superposed objects has the same unit transmission as the single object.

(ii) Linear superposition of the complex amplitudes of the spatial spectra $a(x, y)$ of two or more objects occurs, but of course, the photomultiplier converts only intensities.

(iii) The coefficients $\left|c_{n}\right|$ are not linear functions of the signal $U(t)$, but depend on the relative phases of the components.

These arguments do not support the linear superposition of the components suggested in expression 2. Nevertheless, further detailed study of the problem has shown that in many cases recognition of compound objects, using the method described above leads to satisfactory results. An analysis of 53 clusters

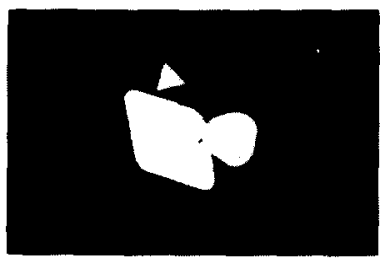

a

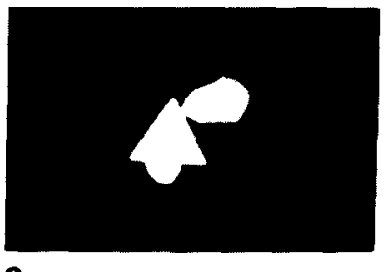

c

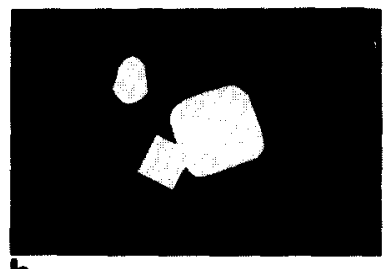

b

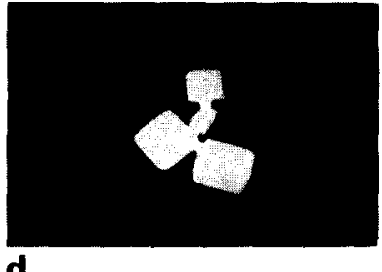

d
Fig. 7 Representative samples of clusters 
has been carried out. Representative samples which were recognized are shown in Fig. 7 . The dominant recognition factor was unequivocal in 41 cases and the dominant objects were clearly detected. Only 12 samples were doubtful, giving either no unequivocal identification or leading to wrong conclusion.

\section{CONCLUSIONS}

The work presented leads to an automatic pattern recognition system, which may, however, only be applied to specific geometrical objects differing only in their two-dimensional rotational symmetry group such as the crystals described. The desired invariance properties are implicit in the method without the special precautions necessary for the classical filtering methods. Furthermore, the method is fastwhich is an important feature. The reliability for single objects was found to be almost $100 \%$ and, for clusters, more than $75 \%$.

\section{ACKNOWLEDGEMENTS}

The authors would like to thank the director of the Institute of Technical Physics and the department of Industrial Research at the ETH, Prof. Dr E. Baumann, for encouragement and support of the work. Thanks are further due to Ciba, Fribourg, for the preparation of the test object shown in Fig. 1.

\section{REFERENCES}

1 Vander Lugt, A. A review of optical data processing techniques. Optica Acta, vol. 1. 1968. pp. 1-33.

2 Armitage, J. D. \& Lohmann, A. W. Character recognition by incoherent spatial filtering. Appl. Opt. vol. 4. 1965. pp. 461-67.

3 Vander Lugt, A. Operational notation for the analysis and synthesis of optical data-processing systems. Proc. IEEE. vol. 8. 1966. pp. 1055-63.

4 Born, M.\& Wolf, E. Principles of optics. 1965.

5 Hannes, $H$. Interferometrische Messungen an Phasenstrukturen fur die Holographie. Optik. vol. 26. 1967. pp. 363-80.

\section{APPENDIX}

Symmetry of the spectrum of the triangle In general, the Fourier transform of a real function $\mathrm{A}(\mathrm{u}, \mathrm{v})$ is

$$
\begin{aligned}
& \operatorname{FT}[A(u, v)]=a(x, y)=a^{*}(-x,-y) \\
& \text { and }|a(x, y)|^{2}=i(x, y)=i(-x,-y)
\end{aligned}
$$
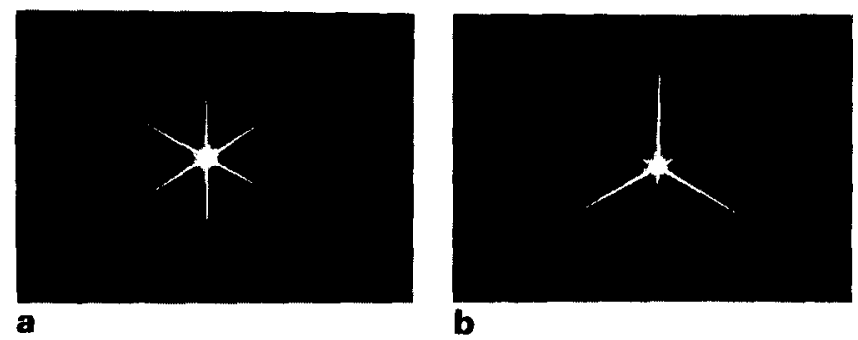

Fig. 8 Spectra of triangles with and without induced phase modulation:

(a) is spectrum of a triangle punched into metal foil showing six-fold symmetry

(b) is a triangle copied on typolith showing threefold symmetry

The intensity spectrum of a real function is always symmetrical as shown in Eqn 3. Hence, the spectrum of a real triangle has the sixfold symmetry shown in Fig. 8a. A triangle punched into a disc was used as object. By contrast, in Fig. 8b, the transparency of the object, a photographic film (typolith), is no longer real. It can be shown that a phase change due principally to a surface swelling near the edge of the transparency object caused a phase modulation of the light and, hence, leads to a threefold symmetry of the spectrum as shown in Fig. 3 and $8 \mathrm{~b}$. The width of the emulsion swelling was found to be about $50 \mu \mathrm{m}$ for objects of $0.5-5.0 \mathrm{~mm}$, and an increase of the thickness of about $300 \mathrm{~nm}$ occurred ${ }^{5}$. This investigation will be subject of a further publication.

Size invariance of the function $\mathrm{e}(\Phi)$ From $A(u, v) \stackrel{\text { FT }}{\rightarrow} a(x, y)$ it follows that

$\overline{\mathrm{A}}(\mathrm{u}, \mathrm{v})=\mathrm{A}(\mathrm{cu}, \mathrm{cv}) \stackrel{\mathrm{FT}}{\rightarrow} \overline{\mathrm{a}}(\mathrm{x}, \mathrm{y})=\mathrm{c}^{-2} \mathrm{a}\left(\frac{\mathrm{x}}{\mathrm{c}}, \frac{\mathrm{y}}{\mathrm{c}}\right)$.

Substitution in Eqn 1 gives

$$
\overline{\mathrm{e}}(\Phi)=\mathrm{e}(\Phi) \cdot \mathrm{c}^{-2}
$$

Changing the upper limit of integration from $\infty$ to $r_{1} \gg r_{0}$ does not appreciably change $e(\Phi)$ for as $\mathrm{r} \rightarrow \infty,|\mathrm{a}(\mathrm{x}, \mathrm{y})|^{2} \approx 0\left[1 / \mathrm{r}^{2}\right]$. By contrast, however, the lower limit of integration $r_{0}$ reduces the low frequency content of $e(\Phi)$ which is, of course, more important for larger objects (small zero order spectrum). The suppression of the low frequencies is largely compensated by the increase of intensity in 3 the spectrum due to the larger object. 